\title{
KINETIC ROSETTE PATTERNS AND TESSELLATIONS
}

\author{
VALENTINA BEATINI \\ Department of Architecture, Abdullah Gül University, Turkey.
}

\begin{abstract}
The paper investigates the possibility to create kinetic rosette patterns and their tessellations by means of modular linkages which rely on the same type and number of symmetry operations as the reference models. The mechanisms show a hierarchy of movements. It is found that symmetry is an effective unifying concept in the design of both the fixed models and the mechanisms. Furthermore, the resulting rosette linkages and their tessellations have peculiar kinematic characteristics if compared to other modular mechanisms which may be alternatively used to reproduce the same kind of models.
\end{abstract}

Keywords: 2D-point symmetry, assemblage of linkages, dihedral and cyclic symmetry, rosette pattern, kinetic tessellation.

\section{INTRODUCTION}

This paper investigates the possibility to create kinetic linkages from rosette patterns.

Geometrically, any rosette is obtained by rotating and in case reflecting a fundamental domain, a minimal generating pattern, around a fixed point. More strictly, any rosette pattern is the result of $2 D$ point symmetry operations.

Symmetry has a key role also in the construction of the proposed kinetic rosettes. In fact, the arrangement of the links and joints that create the mechanism directly derives from the symmetry operations which define the reference rosette. Such strict base on point symmetries gives to the mechanism peculiar kinematic characteristics if compared to other more common modular linkages. Among others, it maintains a fixed center of rotation during motion, and it allows high freedom in setting the dimension and shape of links.

It could be pointed out that rosettes appear in a wide variety of designs. Indeed, it is possible both to vary the type and order of point symmetry operations, as illustrated in Kaplan [1] and Çolakoğlu, [2], and to modify the profile comprised within the fundamental unit, as exemplified in Hankin [3] and Lee [4]. Whatever complex may eventually look the rosette, it had always been constructed and it can always be read by means of simple point symmetry operations. Similarly, the complexity of the reference pattern does not influence the construction and the motion of the proposed mechanism.

In art, single rosettes are often combined into tessellations. Geometrically, there is not a unique classification criterion of rosette tiling. The one proposed by Lee seems especially in line with the definition of the single rosette. Indeed, he considers the entire single rosette as a primitive cell and he focuses on plane symmetry operations to which it is subjected [5]. The same procedure is used here to assembly the rosette linkages. As before, all elements which are irrelevant to the definition of the rosette and of the lattice are irrelevant to the definition of the rosette linkages and of the whole mechanism. Interestingly, tiling offers new kinematic opportunities: the movement of the whole mechanism is determined by the hierarchy and order of symmetry operations which describe the original tessellation. Indeed, as one can follow alternative paths to cover the plane by means of translational symmetry, similarly one can choose to connect the single kinetic rosettes with different sequences. The final design will be the same in all cases. Nevertheless, the mechanism will fold in different ways, and so it will reach different fully folded configurations. 
As reported in Owen [6], rosette patterns have been generating interest for a very long time. Today, historians and technologists discuss on which ingenious techniques old handcrafters might had used to construct them; meanwhile, artists and architects use these patterns as reference models for striking designs. The proposed mechanisms may disclose new possible uses of these decorative patterns and may help classifying rosette tiling. Furthermore, this study remarks once more the importance of symmetry as a unifying concept between different fields. While this paper takes as reference artistic rosettes, the peculiar movement of the modular mechanism may be used in chemistry, mechanical engineering, space structures, and other applications that are not envisioned here.

\section{THE MECHANISM}

Geometrically, rosettes are given by point symmetry operations on a fundamental domain, Fig.1a. The fundamental domain, which is the minimal generating shape, is subjected to $n$ point symmetry operations, where $n$ is called the order of the rosette. As the rosette encompasses the repetition of a fundamental domain, the mechanism is composed of the repetition of a base linkage. The linkage captures the essential characteristic shared by any rosette because it is symmetrical around lines passing through the center. In particular, the linkage reproduces the shapes within four fundamental domains symmetrical around the center (two in case of the trivial rosette of order one), Fig.1b.

Kinematically, since the four fundamental units are doubly symmetrical, the linkage is formed by four doubly symmetrical links, connected by pin joints. Linkages composed by four links and four pin joints, $4 R$-linkages, have one degree of freedom, which means that their movement is completely defined once known the rotation of an input angle. The linkage proposed here, additionally, can fold completely flat because of its double symmetry.

The complete rosette is defined by $n$ symmetry operations, where $n$ is called the order of the rosette. The same number and type of symmetry operations are performed to complete the kinetic rosette, Fig.1c. Additionally, two pin joints connect each linkage with at least one of its adjacent ones until an open chain is formed. Incidentally, we point out that a closed chain would produce a rigid structure and not a mechanism. The connecting joints can be positioned at any intersecting point between the linkages, and comply with the cyclic or dihedral symmetry of the pattern. Nevertheless, if connected linkages are topologically mirror images of each other, all linkages, i.e. all petals, reach a fully folded configuration at the same time regardless of the number of petals, Fig. 1d. Otherwise, a linkage reaches a fully folded configuration when the others are still unfolded.

A closer look at the mechanism highlights this behavior, Fig. 2. Two linkages $A_{0}-E$ and $I-M$ are connected at joints $F_{0}$ and $G$. Topologically correspondent links are labeled with the

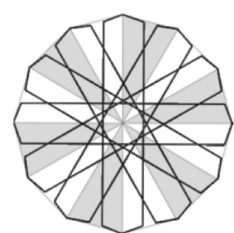

a)

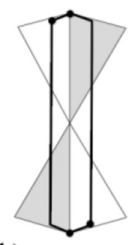

b)

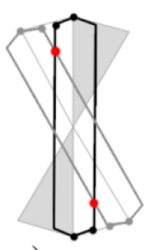

c)

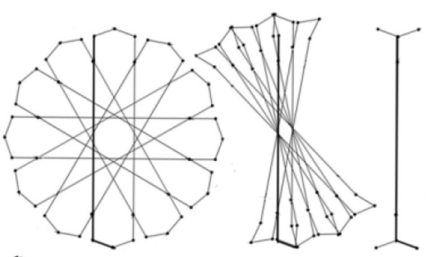

d)

Figure 1: (a) A rosette and its fundamental units. (b) A composing linkage superimposed to its fundamental units. (c) Two interconnected linkages symmetric to each other. (d) The complete kinetic rosette at different folding phases. 
same lowercase letters. The position of any point in the linkages can be expressed by a vector closed loop equation. The vectors which represent the position of the links to the right of the point are put in equation with the ones to the left. The position of point $E$ belonging to linkage $A_{0}-E$ can be defined by

$$
F_{0} G+G D+D E=F_{0} B_{0}+B_{0} E
$$

With respect to a local reference frame $x y z$, eqn (1) in rectangular form reads

$$
d\left(\cos \varepsilon_{1}^{\prime} i^{\prime}+\sin \varepsilon_{1}^{\prime} j^{\prime}\right)+b_{1} j^{\prime}+c_{1}\left(\cos \gamma_{1}^{\prime} i^{\prime}+\sin \gamma_{1}^{\prime} j^{\prime}\right)=a_{1} j^{\prime}+c_{1}\left(\cos \alpha_{1}^{\prime} i^{\prime}+\sin \alpha_{1}^{\prime} j^{\prime}\right) .
$$

Considering linkage $I-M$, the motion of point $L$, which it topologically equivalent to point $E$, is described with respect to a local reference system $x^{\prime} y^{\prime} z^{\prime}$ by the correspondent closed loop equation

$$
F_{0} G+G M+M L=F_{0} K+K L .
$$

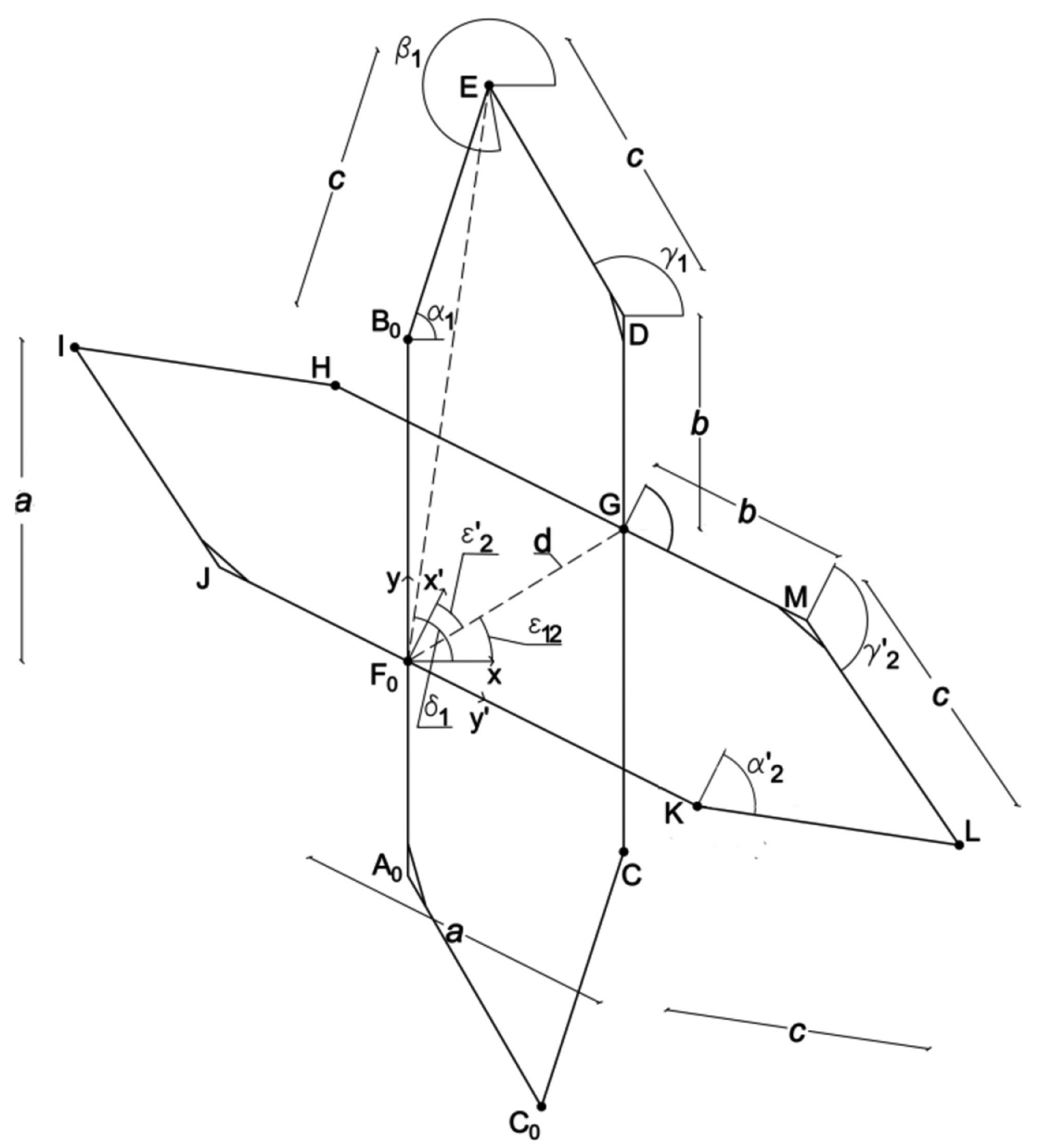

Figure 2: Two petals and their underlying linkages. 
The equation in rectangular form, besides indexes, results equal to (2):

$$
d\left(\cos \varepsilon_{2}^{\prime} i^{\prime}+\sin \varepsilon_{2}^{\prime} j^{\prime}\right)+b_{2} j^{\prime}+c_{2}\left(\cos \gamma_{2}^{\prime} i^{\prime}+\sin \gamma_{2}^{\prime} j^{\prime}\right)=a_{2} j^{\prime}+c_{2}\left(\cos \alpha_{2}^{\prime} i^{\prime}+\sin a_{2}^{\prime} j^{\prime}\right) .
$$

Separating the vertical and horizontal components in eqn (4), it is:

$$
\left\{\begin{array}{l}
d \cdot \sin \varepsilon_{2}^{\prime}+b_{2}+c_{2} \cdot \sin \gamma_{2}^{\prime}=a_{2}+c_{2} \cdot \sin \alpha_{2}^{\prime} \\
d \cdot \cos \varepsilon_{2}^{\prime}+c_{2} \cdot \cos \gamma_{2}^{\prime}=c_{2} \cdot \cos \alpha_{2}^{\prime} .
\end{array}\right.
$$

Squaring and combining one gets

$$
\alpha_{i}=-\varepsilon_{2}+\cos ^{-1}\left(\frac{\left(a_{i}-b_{i}\right)^{2}-2 c_{i}\left(a_{i}-b_{i}\right) \sin \gamma_{i}}{2 c_{i} d}\right),
$$

Again, the same equations could be derived from eqn (2). Since $e_{i}$ and $d$ are shared between two interconnected linkages 1 and 2 , while the links of the two linkages are equal by construction, angle $a_{i}$, is the only variable. Thus, angle $a_{i}$, read in local coordinates, must have the same value in both the interconnected linkages.

Similar equations may be written to describe the position of any other point at any folding phase. In all cases, it can be derived that the only difference between the positions of two correspondent points belonging to the two linkages is given by the angle by which their local reference systems are rotated with respect to each other, Fig. 3. There is thus a hierarchy of movements: each composing linkage folds until it reaches a fully folded state; meanwhile, the entire rosette also rotates until all of its linkages align with the fix link. This behavior would not have occurred if the mirroring operation had not been applied. As a proof, one can imagine to invert the position of an angulated link $\vec{a}+\vec{b}+\vec{c}$ from $H M L$ to $J K L$ : one would need to invert angles $a$ and $g$ in eqn (4), thus breaking the correspondence between the two connected linkages. Without going further, it can be derived that such linkages would not fold and unfold harmoniously.

Summarizing, any rosette pattern can be constructed by means of doubly symmetrical $4 R$-linkages joined together by joints point symmetrically arranged.

Furthermore, until any two interconnected linkages have internal joints mirrored with respect to each other, the linkages will reach a fully folded configuration at the same time.

As mentioned in the introduction, the visual complexity of the shape may have practical implications, but it is irrelevant to the definition of the rosette. The same holds for kinetic rosettes. A fundamental domain depicting a complex shape can still be reproduced by links made of joined elements, which simply copy the fundamental domain. The movement of the linkage stays unaf-

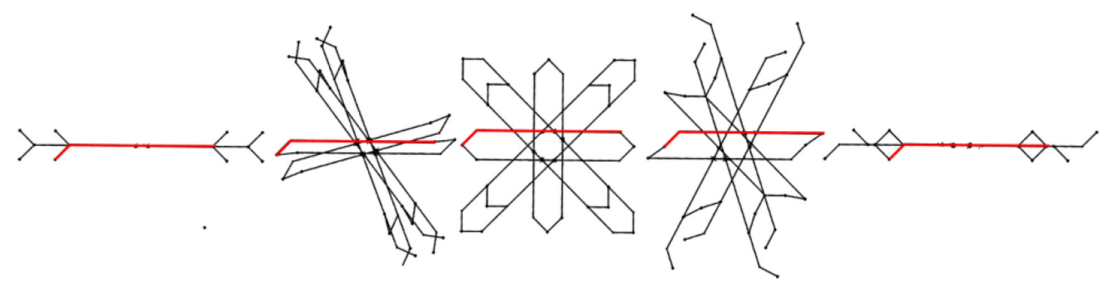

Figure 3: A rosette mechanism based on dihedral symmetry folds harmoniously. 



a)


b)
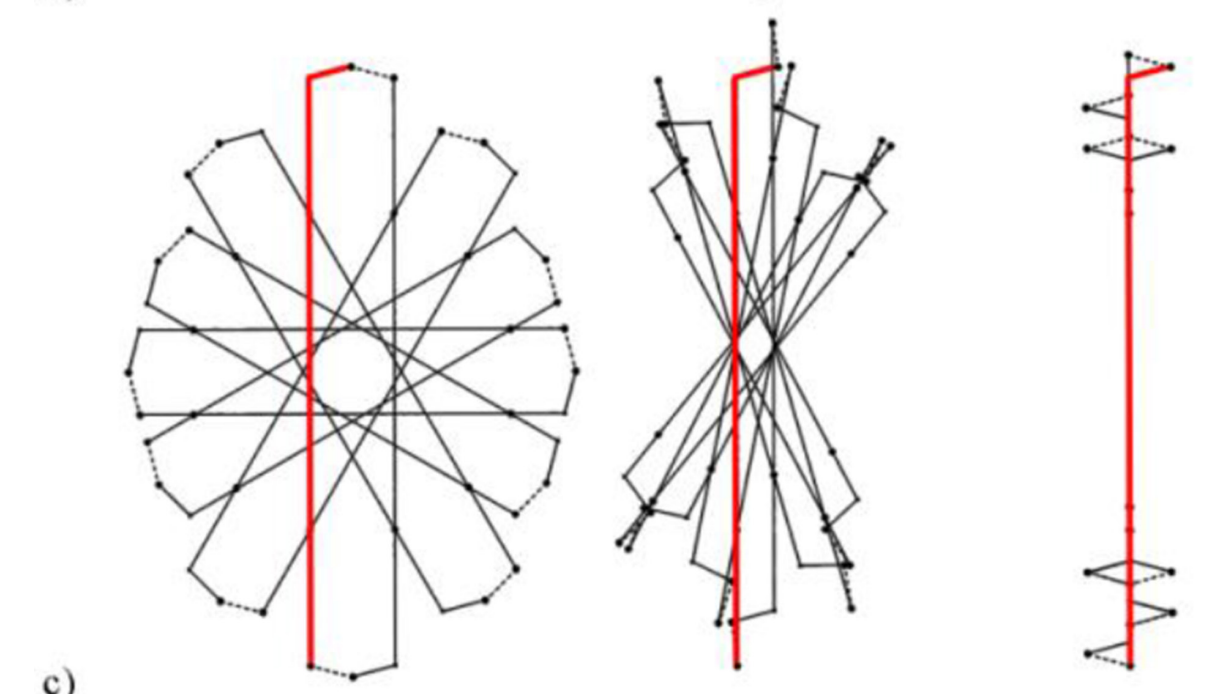

Figure 4: Reference patterns sharing a kind and order of symmetry are constructed with kinematically equivalent linkages. 
fected. Indeed, the movement of linkages is defined by the topology of their joints: their type, their relative distance and their order. As an example, the rosette illustrated in Fig.4a is an aesthetical variation of the one in Fig. 1. Under the same reasoning, other variations can be accomplished.

Some rosettes show a superimposition of symmetry operations, Fig. $4 \mathrm{~b}$ and c. Since in any case there is a partial pattern that is symmetrically repeated in all the rosette, that shared pattern can be used to host the joints connecting linkages to each other. Meanwhile, local variations within each fundamental domain are kept local. They can thus be addressed as usual by modifying the shape of the angulated links within each linkage. Indeed, from eqn (6), with labels referring again to Fig. 2, harmonious folding is still guaranteed with

$$
\begin{aligned}
& a_{1}-b_{1}=a_{2}-b_{2} \\
& c_{1}=c_{2} .
\end{aligned}
$$

\section{TESSELLATIONS}

Two rosettes can be connected to one another by two added pin joints connecting one petal of each. The couple of added joints guarantees that the whole assembly moves with one degree of freedom, because they constrain the two connected $4 R$-linkage to share two links, Fig. 5. These connections are used to reproduce tessellations of rosettes. Similarly, more rosettes can be interconnected. The only constraint is that the added joints create tree-like branches of rosettes without closed loops. As mentioned regarding the single rosette, a closed loop would indeed produce a rigid structure and not a mechanism.

Rosette tessellations can look very complex. Again, it is noteworthy to distinguish between the aesthetical appearance and the geometrical description. Since each rosette is inscribed into a circle, tiling produces interstitial spaces which may be variously filled, Fig. 5. The possible filling segments, which can have virtually any shape, are simply integrated into the shape of the existing links. As discussed concerning the shape of the fundamental domain, until they are rigidly connected, they do not influence the kinematic behavior of the mechanism.

Although there is not a unique definition of rosette tiling, probably the most generic and essential one is in Lee [5]: a tessellation of rosettes is a regular tiling of the plane by primitive cells, here the rosettes, so that a continuous connection between them is achieved. Thus, rosettes can be subjected to any of the translation, reflection and glide reflection plane symmetry operations. In the following images, a rosette of order 4 , i.e. with four lines of symmetry, is subjected to glide reflection, $R x=0$ o $T(0,1)$, Fig. 6, translation along the $x$ and $y$ directions, $T(1,0)$ and $T(0,1)$, Fig. 7, and reflection over direction $y$ and $x, R x=0$ o $R y=0$, Fig. 8 .

Despite the great variety of fully folded configurations which can be obtained even from these simple tessellations, one may recognize the hierarchy of symmetry-based folding rules. They are described here by means of four propositions.



Figure 5: Folding phases of a two-rosette pattern where interstitial segmentsare integrated into the existing links. 

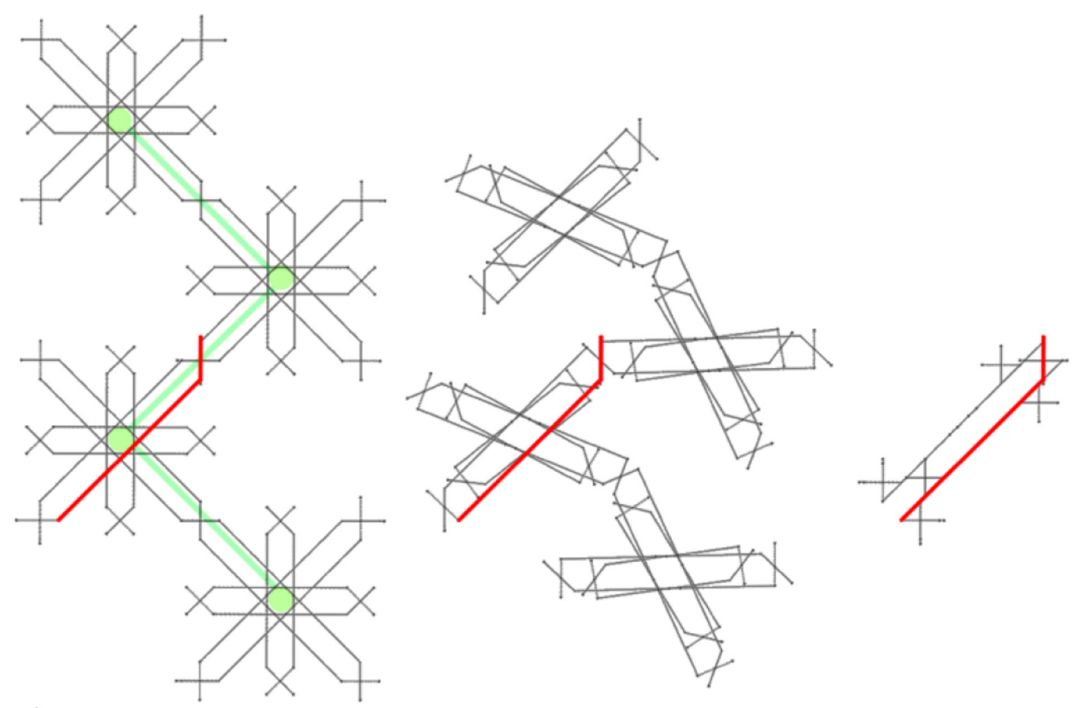

a)
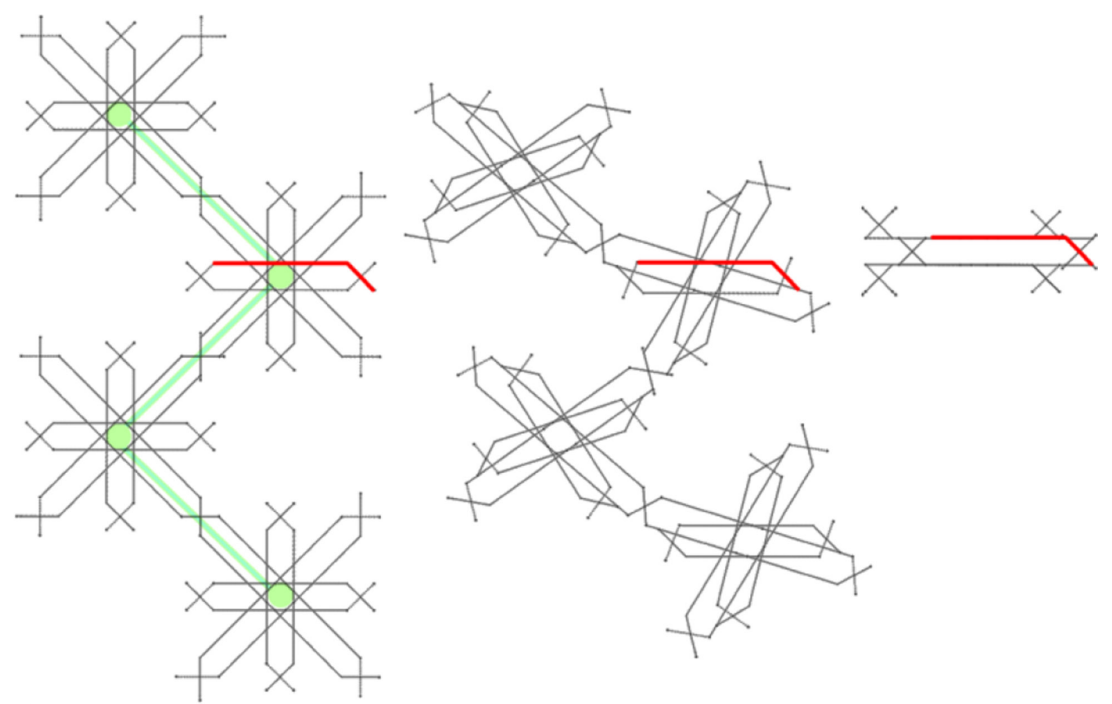

b)

Figure 6: Folding sequences of a mechanism based on glide reflections of a reference rosette. In green, the sequence of connections between adjacent rosettes. In red, the fix link. Changing the fixed link affects the global orientation of the whole mechanism in the fully folded position. The effect is illustrated comparing two otherwise equal tessellations (a) and (b).

- Each kinetic rosette is an assembly of $4 R$-doubly symmetrical linkages. Kinematically, all $4 R$-linkages fold to a fully folded state where all links are coincident.

- Each kinetic rosette, specifically, is a symmetrical assembly of $4 R$-doubly symmetrical linkages. Kinematically, each rosette keeps folding by itself until all its linkages are coincident in the fully folded position. 
- Each rosette is also part of a branch of rosettes related by symmetry operations, so that any two interconnected rosettes share a $4 R$-doubly symmetrical linkage. During motion, each folding rosette as a whole aligns with the two connecting links coming from the previous rosette in the branch, until they become all parallel in the fully folded position.

- The branch of rosette as a whole is connected to the rosette containing the fix link. The branch thus rotates to align with the starting rosette, and ultimately, for the first proposition, to the fix link.

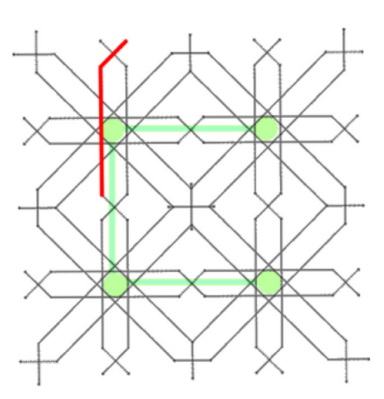

a)

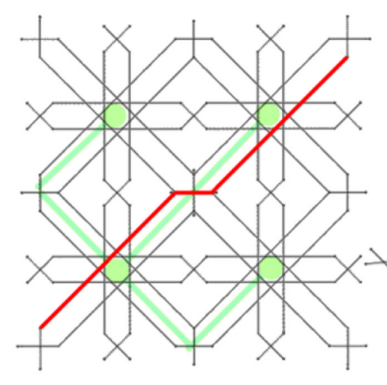

b)

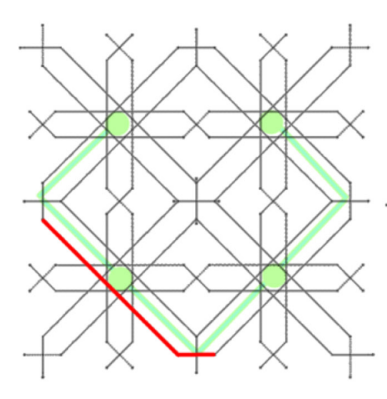

c)
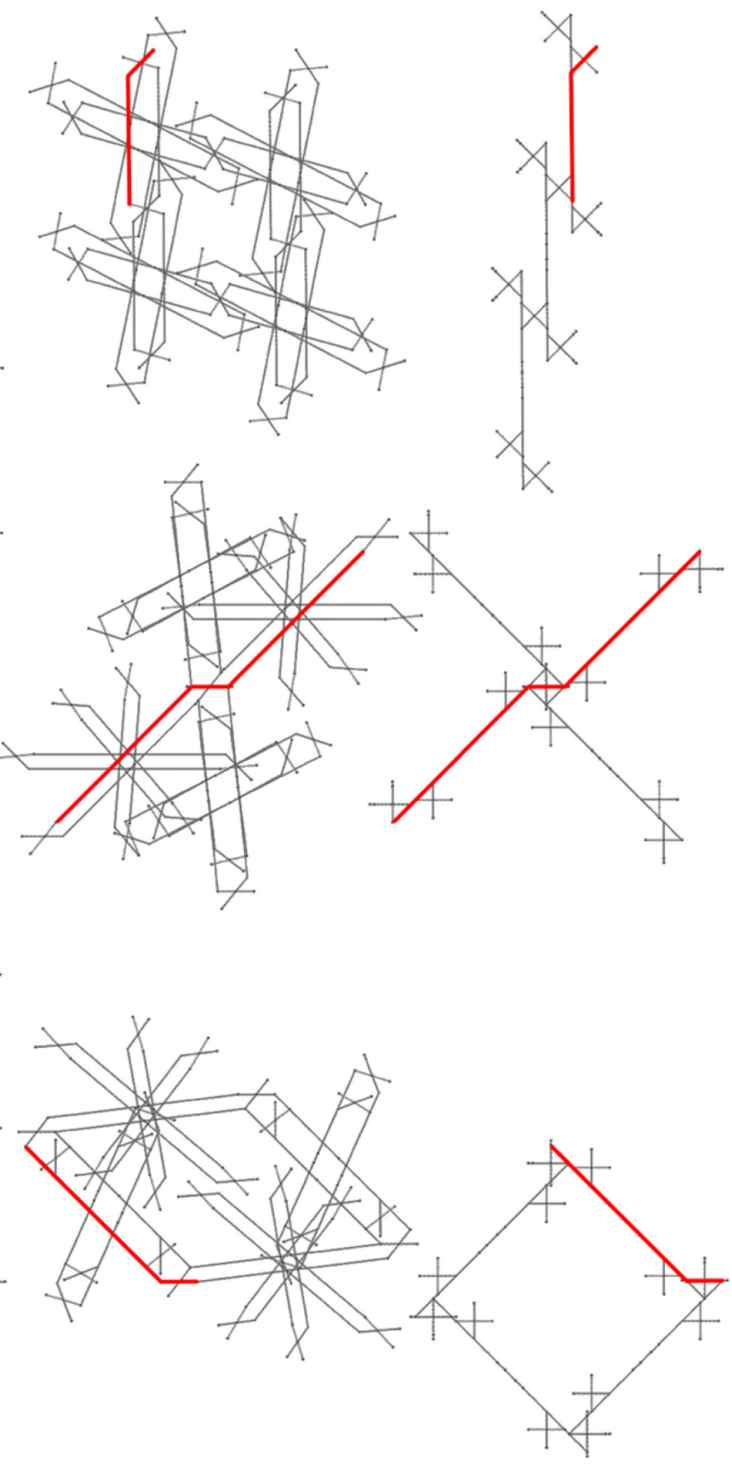

Figure 7: Folding sequences of a mechanism based on translation of a reference rosette. In green, the sequence of connections between adjacent rosettes. In red, the fix link. While the linkages within each rosette fold with respect to their common center, they align with the connected linkage from the precedent rosette in the branch. Changing the tree of connections affects the reciprocal orientation of rosettes during motion. The effect is illustrated comparing three otherwise equal tessellations, (a), (b) and (c). 




a)
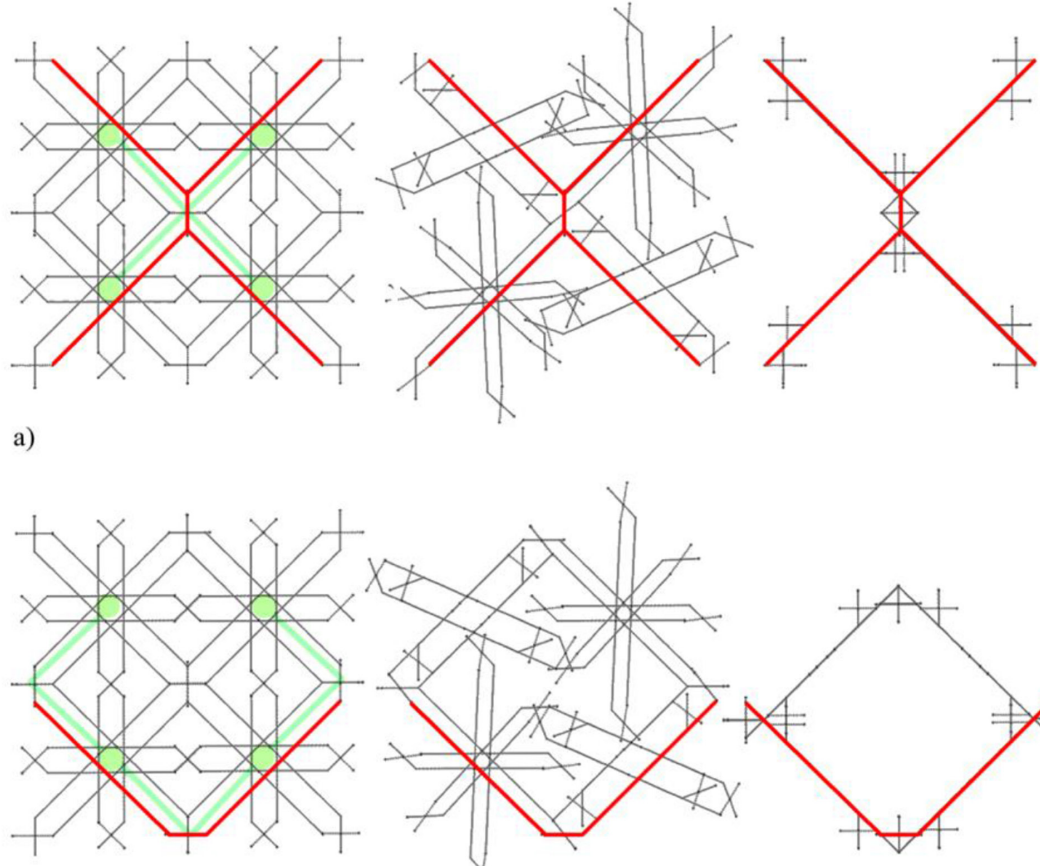

b)



b)

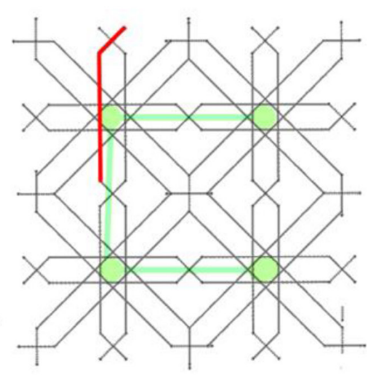

c)

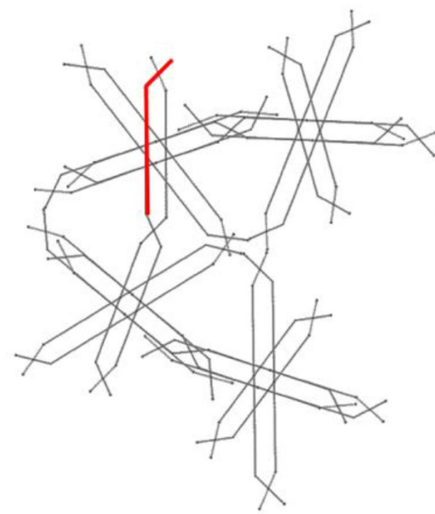

Figure 8: Folding sequences of a mechanism based on mirror of a reference rosette. In green, the sequence of connections between adjacent rosettes. In red, the fix link. The same graphs of connections as in Fig. 5 produce different fully folded profiles thanks to the inverted position of the angulated links within adjacent rosettes. Three alternative branches of connections (a), (b) and (c) are depicted.

The above-mentioned propositions are the rules behind the folding process of these mechanisms. The variety of achievable movements, exemplified in the following pictures, depend on two variables. First, since links can be angulated, i.e. formed by two or more segments rigidly connected at some angle, when the mechanism is fully folded links may be aligned along any of their composing segments. Second, there may be alternative orders into which perform the symmetry operations underlying the tessellations, and thus different connection sequences between the rosettes. Once aware of the rules and of the variables, one may exploit the design possibilities offered by the proposed mechanisms. 


\section{DISCUSSION AND CONCLUSION}

The paper investigated the analogies between rosette symmetries and $4 R$-linkages. The study had been applied to stand-alone rosettes and to their planar tessellations.

Both the mechanism and the reference model are defined only by symmetry operations. Any other element which affects the aesthetical appearance is irrelevant to the definition of the reference model and to the movement of the mechanism. In all cases, the mechanism folds harmoniously with one degree of freedom.

Stand-alone kinetic rosettes are created by means of symmetrical assemblies of $4 R$-doubly symmetrical linkages. Thanks to its inner symmetry, each composing linkage can fold fully independently from the shape of the links, which can be composed by more segments rigidly jointed together. Meanwhile, the symmetrical connections between the composing linkages facilitate construction. On one side, assuming that linkages move on parallel planes, each one can be connected simply to the adjacent ones. On the other side, the center of rotation is fixed during motion, thus a supporting structure may be easily inserted. A simple cardboard model of the rosette is depicted in Fig. 9. While there exist other mechanisms which can produce rosette symmetries in the fully unfolded state, they are either much more complex (iris mechanism), or they are difficult to be organized into assemblies [7, 8].

Furthermore, until any two interconnected linkages have internal joints mirrored with respect to each other, the linkages will reach a fully folded configuration at the same time. One can thus recognize a hierarchy of movements: each composing linkage folds until all its links coincide, and then the linkages all together fold to align with the fix link.

Kinetic tessellations are straightforwardly assembled again following the symmetry rules behind the reference model. They add another level to the hierarchy of movements: not only the basic linkages within each rosette and the entire rosette fold until all the links are coincident, but also the branches of interconnected rosettes align with the fix link of the overall mechanism. Since links are angulated, and since there may be alternative paths of connections between rosettes, many alternative motions can be designed, each producing a substantially different folded configuration.

Despite their long history, both rosettes and $4 R$-linkages are still a fascinating subject to mathematicians, designers, engineers and more. Highlighting their analogies may open new opportunities for their application.

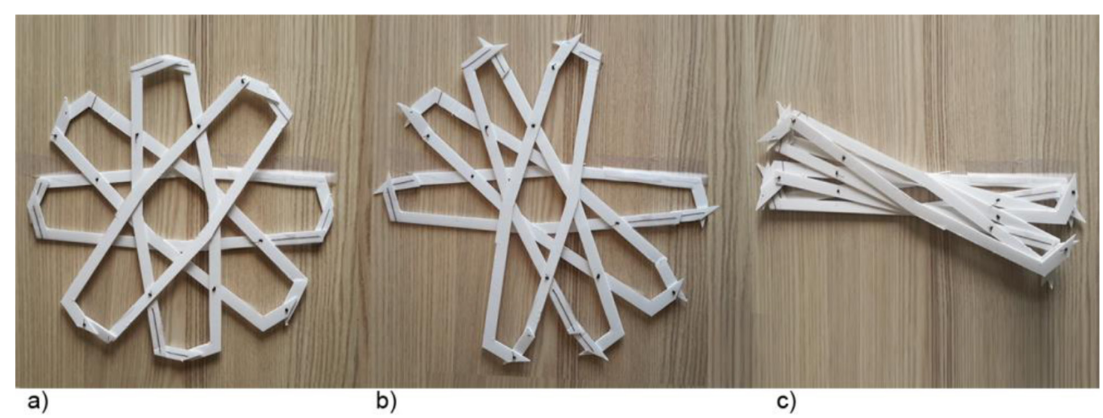

Figure 9: Cardboard model of the rosette already depicted in Fig. 4a. By joining links one upon the other, there is no risk of intersection between them. 


\section{REFERENCES}

[1] Kaplan, C.S. \& Salesin, D.H., Islamic star patterns in absolute geometry. ACM Transactions on Graphics, 23(2), pp. 97-119, 2004.

http://dx.doi.org/10.1145/990002.990003

[2] Çolakoğlu, B., Yazar, T. \& Uysal, S., Educational experiment on generative tool development in architecture. PatGen: Islamic star pattern generator. Proceeding 26th eCAADe Conference. Education and Research in Computer Aided Architectural Design in Europe, eCAADe, 2008.

[3] Hankin, E.H., The drawing of geometric patterns in Saracenic art. Memoirs of the Archaeological Society of India, 15, 1925.

[4] Lee, A.J., Islamic Star Patterns. Muqarnas, 4, pp. 182-197, 1995.

[5] Abbas, S.J. \& Salman, A., Symmetries of Islamic Geometrical Patterns, World Scientific: Singapore, 1995.

http://dx.doi.org/10.2307/1523103

[6] Owen, J., The Grammar of Ornament, Dover Publications: New York, 1989.

[7] Luo, Y., Mao, D. \& You, Z., On a type of radially retractable plate structures. International Journal of Solids Structures, 44(10), pp. 3452-3467, 2007.

http://dx.doi.org/10.1016/j.ijsolstr.2006.09.035

[8] Beatini, V., Kinetic planar tessellations. Proceedings of the International Association for Shell and Spatial Structures, Future Visions, IASS, Amsterdam, 2015. 